\title{
EL SISTEMA APÍCOLA EN LAS REGIONES I Y II DE YUCATÁN: UN CAMBIO DE PARADIGMA EN LAS REGLAS, POLÍTICAS Y RELACIONES
}

\section{THE BEEKEEPING SYSTEM IN REGION I AND II OF YUCATAN: A PARADIGM SHIFT IN THE RULES, POLICIES AND RELATIONSHIPS}

Reception: 2019-12-11 - Acceptance 2020-05-23

\section{Joaquín Eduardo Franco Navarrete ${ }^{1 \text {; }}$}

(iD) ORCID iD 0000-0002-3235-0983

\author{
Mayanín Asunción Sosa Alcaraz ${ }^{1 ; ~ b}$ \\ (iD) ORCID iD 0000-0003-4237-9453 \\ ${ }^{1}$ Tecnológico Nacional de México Campus Instituto Tecnológico de Mérida. \\ a Ingeniero en Administración. \\ b Doctora en medio ambiente y sustentabilidad.
}

\section{RESUMEN}

En Yucatán se producen entre diez mil y quince mil toneladas de miel al año; de esta derrama económica dependen más de once mil apicultores maya hablantes de zonas rurales. Sin embargo, la inexistencia de un Sistema Producto Apícola fortalecido ocasiona que no exista sinergia entre los actores de este sistema. Esto provoca un débil sistema incapaz de afrontar amenazas externas latentes que podrían causar la extinción de las abejas, poniendo en peligro otras actividades como la agricultura, ganadería y forestería. En este artículo, presentado en el 6to Congreso Internacional de Sustentabilidad 2019 Sede México, se muestra una investigación cualitativa sobre la incipiente existencia del Sistema Producto Apícola en dos regiones diferentes del Estado de Yucatán; el objetivo principal es analizar la situación actual de la apicultura en dos municipios de Yucatán, pertenecientes a la región I denominada "Poniente" y región II denominada "Noroeste", los actores clave que conforman dicho sistema y cómo están preparados para combatir amenazas externas/internas. Resultados preliminares obtenidos a través de una revisión documental superficial demuestran la falta de políticas encaminadas a fortalecer los cultivos agroecológicos sobre los convencionales, la amenaza de la introducción de cultivos transgénicos a la región y la ausencia de alianzas estratégicas.

Palabras clave: Apicultura; Yucatán; Alianzas estratégicas.

\section{ABSTRACT}

In Yucatan it is produced between ten thousand and fifteen thousand tons of honey per year; more than eleven thousand Mayan-speaking beekeepers in rural areas depend on the economic flow from this income. However, due the absence of an organized incentive program for apiculture, there is no synergy between the components of this system. This results in a weak system unable to face latent external threats that could cause the extinction of bees, jeopardizing other income-producing activities such as agriculture, cattle industry and forestry. In this article, presented at the 6th International Sustainability Congress 2019, in México, is shown qualitative research on the emerging existence of Apicultural Product System in two regions of Yucatan. The main objective is to analyze the current situation of beekeeping in two municipalities of Yucatan, belonging to the western and northwestern region, the key actors that are members of this system, analyzing their level of preparation and capacity to fight external threats to Yucatecan beekeeping. Preliminary results obtained through the documentary review shows a lack of policies aimed at strengthening agro-ecological over conventional crops and absences of strategic alliances.

Keywords: Beekeeping; Yucatan; Strategic alliances. 


\section{INTRODUCCIÓN}

La apicultura tiene una gran importancia social, económica y ambiental para México; es considerada como una de las principales actividades agropecuarias generadora de divisas y parte fundamental de la economía social en zonas rurales. México se ha consolidado como un gran productor y exportador de miel de gran calidad a nivel global, teniendo como principales destinos los mercados europeos y norteamericano. La Secretaría de Agricultura y Desarrollo Rural (SADER) reportó en el 2019 un incremento en la producción de miel del 6\% respecto a los últimos 10 años. Sin embargo, existen factores como el clima, la competencia desleal, así como el debilitamiento del Sistema Producto Apícola a nivel nacional que lo hace vulnerable (Güemes, Echazarreta, Villanueva, Pat y Gómez, 2003). Actualmente, la apicultura yucateca enfrenta una descoordinación entre los integrantes de la cadena de producción apícola; no se trabaja en equipo para beneficio de la apicultura. Lamentablemente, tanto la Unión de Apicultores y el Comité Apícola de Yucatán mantienen una relación no muy estrecha, como la tenían hace algunos años cuando coordinaban actividades en conjunto; por lo que, trabajar en equipo con los actores clave de la cadena de producción apícola es la única forma de salvar la apicultura. Esta descoordinación no es el único problema que enfrenta la apicultura yucateca; existe una cruzada legal por evitar que la producción de soja transgénica entre al Estado, lo cual afectaría a cientos de productores de miel (Suárez, 2017). Recientemente, el gobierno nacional y estatal ha propuesto políticas y programas públicos para el rescate y manejo sustentable de la apicultura, pero aún falta mucho por hacer.

Por lo arriba expuesto, esta investigación presenta información sobre la debilitada existencia de un Sistema Producto Apícola en dos regiones diferentes del Estado de Yucatán; el objetivo principal es analizar la situación actual de la apicultura en los municipios de Maxcanú y Mérida, pertenecientes a las regiones I Poniente y II Noroeste respectivamente, ambos municipios del Estado de Yucatán. Asimismo, se busca identificar actores clave integrantes de este sistema y examinar cómo está preparado para combatir amenazas externas e internas, a partir de nuevos cambios en la legislación nacional, la falta de alianzas, de capacidades humanas, de innovación, y políticas públicas que puedan fortalecer la cadena productiva de la miel.

De acuerdo al Servicio de Información Agroalimentaria y Pesquera (SIAP, 2020), la península de Yucatán es la región mexicana productora de miel más importante, con una producción promedio de 17370 toneladas por año, y alrededor de 12 mil apicultores dedicados exclusivamente a esta actividad. Esto convierte a la apicultura yucateca en una de las actividades socioeconómicas más importante en zonas rurales del Estado. De hecho, Ayala Arcipreste, experta en ecología humana, considera que el 95\% de la miel que se produce en la región peninsular se encuentra destinada al mercado exterior. Esto puede ser considerado una ventaja e indicador de productividad, pero en algunos casos, tener una producción destinada casi en su totalidad al mercado externo, puede traer graves consecuencias (Suárez, 2017). Un ejemplo de esto es lo que se vive actualmente en la Región Oriente del Estado de Yucatán, en donde las exportaciones, principalmente a Alemania, están detenidas por motivos desconocidos; esto provoca que los centros de acopio y unidades económicas de la región rechacen la compra de miel a pequeños productores. Ante esto, el Gobierno de Yucatán, con el objetivo de reducir el número de intermediarios en la cadena de valor y asegurar la compra de la producción a pequeños productores, ha anunciado la creación de un centro de acopio en la zona oriente (Miranda, 2016). Aunque, tal vez sea más importante elevar la capacidad de comercialización internacional, a través de capacitación técnica y control de calidad; como resultado de esto, se podría vender a mejor precio la miel.

El mercado internacional de la miel se encuentra regido por normas, procesos y dinámicas muy concretas; la oferta y demanda responden muy rigurosamente a elevados estándares de calidad, basados en políticas gubernamentales que en muchas ocasiones provienen de intereses privados y no siempre se encuentran bien argumentados. De hecho, en 2012, un tribunal europeo votó por prohibir la compra-venta de miel que contenga rastros transgénicos. Estas decisiones han derivado en el encarecimiento de ciertos productos, lo que reduce su penetrabilidad para ciertos mercados y pone en desventaja a algunos productores (Güemes, Echazarreta, Villanueva, Pat y Gómez, 2003). Un ejemplo de lo anterior sucedió en Campeche, México con la apertura a los cultivos transgénicos; actualmente se continúa efectuando una batalla legal entre productores de miel orgánica y la multinacional agroalimentaria Monsanto. En este Estado se ha permitido el cultivo de variedades de soya y maíz transgénico, lo que ha contaminado la miel de los apicultores campechanos (Enciso, 2018). Ahora, con la decisión de la Suprema Corte de Justicia de la Nación (en México) de invalidar un decreto en donde se prohibía el cultivo transgénico en Yucatán, se pone en juego el destino de los pequeños apicultores yucatecos (Santana, 2019). Estos productores locales, especialmente de comunidades indígenas, tienen procesos más tradicionales. De acuerdo con Merrill-Sands (1984), la práctica apícola en Yucatán responde a una lógica de subsistencia familiar, diferente al de economía de mercado; esto se traduce en una práctica milenaria heredada por los mayas que tiene como objetivo lograr la seguridad alimentaria antes que la económica. Ante esta problemática, la 
apicultura ha fungido como una "noble" actividad productiva que permite la subsistencia de gran parte de los campesinos de las zonas rurales marginadas, pero para lograr la permanencia de esta actividad, es necesario entender y conocer cómo están formados y organizados los actores claves que participan dentro de la apicultura siendo la mejor manera de lograr esto por medio de los Sistemas Producto y en específico, el Sistema Producto Apícola.

Para darse una idea de lo que es un Sistema Producto, la Secretaría de Agricultura, Ganadería, Desarrollo Rural, Pesca y Alimentación (SAGARPA), en sus políticas de protección y fomento, se encaminó hacia las unidades productivas. Posteriormente se pasó a un sistema integrado donde los productores se agrupan a nivel local, regional o nacional, de manera jerárquica, para facilitar la gestión y toma de decisiones (Cuevas, Baca y Aguilar, 2011).

Por lo anterior, los Sistemas Producto son formas de agrupación, gestión y organización en donde los productores y demás agentes relacionados con la apicultura, se organizan de manera jerárquica y tienen presencia a nivel nacional, estatal y municipal, apegándose a la Ley de Desarrollo Rural Sustentable vigente. Estas representaciones tienen el objetivo de resolver dificultades en la cadena de producción, desde el aspecto productivo, atravesando por la transformación y hasta que se cumple la comercialización, es decir de toda la cadena productiva. Asimismo, estas formas de organización son necesarias para elaborar planes de expansión y estrategias, de acuerdo con las regulaciones, tendencias de mercados y condiciones del país (Secretaría de Desarrollo Agropecuario del Estado de México, 2018).

En México, el fundamento legal del concepto de Sistema Producto se fundamenta en la Ley de Desarrollo Rural Sustentable, artículo tercero, fracción XXXI. Entre los artículos importantes destacan: Art. 149.- La Comisión Intersecretarial promoverá la organización e integración de Sistemas Producto, como Comités del Consejo Mexicano, con la participación de los productores agropecuarios, agroindustriales y comercializadores y sus organizaciones, en beneficio de cada actividad productiva. Artículo 150.- Se establecerá un Comité Nacional de Sistema Producto por cada producto básico o estratégico, teniendo para cada Sistema Producto un solo Comité Nacional, con un representante de la institución responsable. Artículo 151.- Se promoverá la creación de los Comités Regionales de Sistema Producto (Ley de Desarrollo Rural Sustentable, 2001).

El Comité Nacional Sistema Producto Apícola surge por la necesidad de organizar los procesos de autogestión de todos los agentes participantes en la apicultura mexicana, desde pequeños productores hasta los más grandes exportadores de este alimento natural. Uno de los principales objetivos de la organización es la conformación del plan rector para el desarrollo de la actividad apícola en el país, con el objetivo de beneficiar a todos los partícipes en la actividad e involucrarlos de tal forma que se logre desarrollar una apicultura más sustentable e integral. A través de proyectos, se busca impulsar, potenciar y posicionar la apicultura mexicana en el mundo.

En Yucatán, debido a la necesidad de ejecutar políticas gubernamentales encaminadas a aumentar la calidad y productividad, se crea el Comité Sistema Producto Apícola de Yucatán bajo el decreto número 521 publicado el 6 de julio de 2004, teniendo como última reforma publicada el 31 de julio de 2019, en el Diario Oficial del Estado de Yucatán. Entre los artículos más destacados se mencionan: Artículo 2.- El objetivo de esta ley es la potenciación de la apicultura en el Estado; especialmente en áreas rurales idóneas para la apicultura y que pueda representar una actividad económica de arraigo para los pobladores. Artículo 7.- La Secretaría de Desarrollo Rural (SEDER) presidirá el Comité de Sistema Producto Apícola e impulsará la coordinación de éste; asimismo, promoverá y fomentará el registro de apicultores y su organización. Artículo 8.- El principal objetivo del Comité Sistema Producto Apícola del Estado de Yucatán, es integrar a los agentes económicos participantes en la actividad para darle solución a las diversas problemáticas apícolas. Artículo 9.- Habla de los miembros que integrarán el Comité Sistema Producto Apícola de Yucatán (Decreto 521/2004).

Además, el 31 de octubre de 2017, el Gobierno del Estado de Yucatán publicó un decreto sin precedentes, el cual es identificado como Decreto 537/2017 por el que se establecen las zonas apícolas del Estado de Yucatán para el fomento de su aprovechamiento racional. Entre sus estipulados más importantes se pueden encontrar las siguientes consideraciones:

- La actividad apícola está contemplada en más del 80\% de las Unidades de Gestión Ambiental (UGA) del Estado.

- Que la apicultura en la actualidad es amenazada por la presencia de polen de organismos genéticamente modificados, que disminuyen su calidad y su precio a nivel internacional. 
- Que el mercado europeo es muy exigente con respecto a la calidad de la miel y establece restricciones a la comercialización si la miel presenta contaminación de algún tipo, lo que representa pérdidas en las ventas.

- Que la región sureste de México es la más importante de las cinco regiones apícolas del país, tanto por la población rural involucrada en la actividad, como por el volumen y valor económico de la producción (Decreto 537/2017).

\section{MATERIAL Y MÉTODOS}

La investigación se ha desarrollado a través de un estudio de casos, apoyado por revisión documental, de literatura y entrevistas a profundidad. Este trabajo tiene como objetivo presentar información acerca del Sistema Apícola de las regiones I Poniente y II Noroeste del Estado de Yucatán e identificar actores clave integrantes de este sistema. Para ello, fue necesario conocer las percepciones de los diversos actores sobre la problemática, analizar cómo están preparados para combatir amenazas externas e internas a partir de nuevos cambios en la legislación nacional, su falta de alianzas, de capacidades humanas, de innovación y aquellas políticas públicas que estén o no apoyando el buen desarrollo de la cadena productiva de la miel; asimismo, permitirá identificar el marco normativo en materia de protección a esta actividad agropecuaria. De acuerdo con Yin (1989), el estudio de caso es una herramienta de la investigación cualitativa, y su principal ventaja es que mide y registra la conducta de los sujetos de estudio en el fenómeno que se investiga; busca entender el fenómeno y conocer cómo es que éste se da, así como las partes que lo componen y las relaciones existentes entre ellas. Para esta investigación, el Sistema Producto Apícola y su situación actual en las regiones I Poniente y II Noroeste del Estado de Yucatán comprenden el estudio de caso.

Para ello, se utilizó el método de muestreo denominado "bola de nieve", en donde se identifican sujetos de interés o actores clave, a través de una "red de conocidos" que pueden resultar buenos candidatos para participar en la investigación (Martínez-Salgado, 2012). Fue una muestra intencional, ya que se decidió entrevistar a personas que desempeñan un papel clave en el estudio de caso (Yin, 1989), en particular el Sistema Producto Apícola. Como resultado, se identificaron y entrevistaron a 8 sujetos de estudio involucrados en el Sistema Producto Apícola de las regiones I Poniente y II Noroeste de Yucatán, que de manera voluntaria accedieron a proporcionar información a través de entrevistas a profundidad. Entre ellos se encuentran: a) tres apicultores de Maxcanú, Yucatán; b) un representante del Comité Nacional Sistema Producto Apícola, c) un representante gubernamental de Yucatán, d) un académico, e) un experto técnico y f) un empresario exportador. Por razones de seguridad, conflicto de intereses y a petición de los entrevistados, serán identificados con claves. Todos los sujetos de estudio se ubican en los Municipios de Maxcanú y Mérida, de las regiones I Poniente y II Noroeste respectivamente, ambos municipios del Estado de Yucatán. La entrevista consistió en conversar con los actores clave de manera abierta sobre la existencia y funcionamiento del Sistema Producto Apícola a nivel nacional, estatal y regional, las principales fortalezas que se tienen, así como los retos que están enfrentando para la comercialización de sus productos, los cambios en las legislaciones jurídicas y ambientales, y los múltiples problemas internos que viven día a día.

Para complementar y triangular la información de las entrevistas a profundidad, se llevó a cabo una muestra teórica para reunir más testimonios sobre lo que está ocurriendo dentro del Sistema Apícola en las regiones I Poniente y II Noreste de Yucatán. Esta muestra se realizó a través de una revisión literaria, la cual es una técnica en donde se extrae y recopila información sobre el fenómeno a investigar. A través de ella, se consulta bibliografía y se reúne información relevante y necesaria, concerniente al fenómeno o problema de investigación (Cortés y León, 2004). La revisión literaria para esta investigación consistió en revistas, artículos, notas periodísticas, entre otros documentos de divulgación pública. Entre ellos se encuentran los siguientes.

- Ley de Desarrollo Rural Sustentable, artículo tercero, fracción XXXI; artículos 149 a 153 de la LDRS; Diario Oficial de la Federación, 2001

- Decreto 521/2017 de la Ley De Protección Y Fomento Apícola Del Estado De Yucatán

- Decreto 537/2017 por el que se establecen las zonas apícolas del Estado de Yucatán, para el fomento de su aprovechamiento racional

- Decreto Número 583; Reglamento De La Ley De Protección Y Fomento Apícola Del Estado De Yucatán.

- Medios de circulación regionales, como son periódicos, diarios, revistas, entre otros. 
- Artículos de divulgación agropecuaria de la SADER, así como la SEDER y el Instituto Nacional de Investigaciones Forestales, Agrícolas y Pecuarias.

El análisis de la información se realizó identificando palabras y frases clave que ayuden al entendimiento en relación a los siguientes temas: a) las alianzas que pudieran existir entre los diferentes actores que componen la cadena productiva de la miel, b) los cambios legislativos y ambientales que pueden llegar a afectar a la producción apícola del Estado, y c) los programas y políticas públicas que estén apoyando el fortalecimiento del Sistema Producto Apícola en estas regiones de Yucatán. Por eso, con el propósito de entender la percepción propia de todos y cada uno de los participantes acerca de la problemática y situación existente, se elaboró una tabla en Excel ilustrando el nivel de conocimiento que poseen sobre la situación real del Sistema Apícola, los factores que intervienen en este, el trabajo en conjunto con otros sujetos dentro del mismo sistema y si están vinculados estratégicamente entre ellos.

\section{RESULTADOS}

De acuerdo a las entrevistas a profundidad aplicadas a los actores clave integrantes del Sistema Producto Apícola de las regiones I Poniente y II Noroeste de Yucatán (ver tabla 1), la revisión documental, el análisis de la existencia, integración y funcionamiento actual del sistema en estas regiones, así como del marco normativo y las políticas públicas que lo protegen, se demuestra la inexistencia de una red de actores consolidada, fuerte y abiertamente visible para todos los integrantes de la cadena de producción. Esto pone en entredicho lo dispuesto por la Ley de Protección y Fomento Apícola del Estado de Yucatán, que obliga a la integración de un Comité Sistema Producto Apícola en el Estado (Ley de Protección y Fomento Apícola del Estado de Yucatán, 2004). Esta ley expone la necesidad de contar con una red de actores claves que se integren y trabajen en sinergia para beneficio de la apicultura yucateca; destacando en primera instancia a los productores apícolas, empresarios, emprendedores, expertos técnicos, ambientalistas, académicos y al gobierno que, a través de las políticas públicas y sus dependencias, protegen y cuidan esta actividad productiva de amenazas externas. Los resultados que se muestran a continuación combinan y contrastan la información obtenida por los dos métodos aplicados en este estudio de caso.

Tabla 1

Sujetos de estudio

\begin{tabular}{ccc}
\hline Clave & $\begin{array}{c}\text { Participación en el Sistema Producto } \\
\text { Apícola }\end{array}$ & $\begin{array}{c}\text { Institución u Organización que } \\
\text { pertenece }\end{array}$ \\
\hline Apicultor 1 & Productor & Maxcanú, Yucatán \\
Apicultor 2 & Productor & Maxcanú, Yucatán \\
Apicultor 3 & Productor & Maxcanú, Yucatán \\
$\begin{array}{c}\text { Representante No } \\
\text { Gubernamental del Comité } \\
\text { Nacional SPA }\end{array}$ & Tomador de Decisiones & $\begin{array}{c}\text { Comité Nacional Sistema } \\
\text { Producto Apícola }\end{array}$ \\
$\begin{array}{c}\text { Representante } \\
\text { Gubernamental }\end{array}$ & Servidor Público/Gubernamental \\
Académico & Catedrático/ Academia & $\begin{array}{c}\text { Secretaría de Agricultura y } \\
\text { Desarrollo Rural Yucatán }\end{array}$ \\
Experto Técnico Apícola & Investigador/Sociedad Civil & Tecnológico Nacional de México \\
Empresaria Exportadora & Comercializador/Empresas & Sociedad Civil \\
\hline
\end{tabular}

Fuente: Elaboración propia. 
Entre los principales hallazgos se encontró un incipiente Sistema Producto Apícola en las regiones I Poniente y II Noroeste de Yucatán, con poca o nula integración efectiva entre sus participantes, quienes no se encuentran abiertamente identificados ni trabajan en sinergia entre ellos, provocando un desconocimiento generalizado de las actividades de cada uno de ellos, y entre ellos, lo que limita y obstaculiza la creación de proyectos integrales en favor de la apicultura. Asimismo, se destaca la débil legislación en materia de protección apícola en Yucatán; con leyes, normas y políticas públicas que no fortalecen ni preparan al sector apícola para afrontar amenazas externas venideras, por lo que la actividad está en alto riesgo. De acuerdo al representante No Gubernamental del Comité Nacional Sistema Producto Apícola (sujeto de investigación entrevistado en este estudio), se reconoce oficialmente la existencia de una representación estatal en Yucatán; sin embargo, desde el sexenio pasado, el apoyo a los Sistemas Producto ha sido errático, o peor aún, inexistente. Asimismo, lamenta que el Comité Estatal en Yucatán se ha mantenido trabajando con sus propios medios, lo que limita los avances e innovaciones que se pudieran hacer en pro de la apicultura. Añade que, en la actualidad, el Gobierno Federal está trabajando por la renovación del Consejo Mexicano para el Desarrollo Rural Sustentable, a quien pertenecen los Sistemas Producto, y espera que estos Consejos Estatales vuelvan a tomar su legítimo lugar como consejos consultivos y generadores de información del sector para la mejor toma de decisiones (Representante No Gubernamental del Comité Nacional Sistema Producto Apícola, comunicación personal, 9 de septiembre de 2019). De hecho, ante la preocupante situación que enfrenta la apicultura yucateca, en 2018, un senador del Estado de Yucatán, se reunió con productores y académicos del Estado para la elaboración de un proyecto integral que mejore la situación apícola de todas las regiones de Yucatán; en las intervenciones se plantearon temas como la grave pérdida de floración (como la del tahonal, planta emblemática de la península de Yucatán para la apicultura cuyo nombre científico es viguiera dentata), los problemas de plagas que reducen la producción de néctar, la falta de cera estampada, la necesidad de cambiar cajas y equipo en general, el riesgo de la siembra de transgénicos, el uso de pesticidas y la deforestación de grandes extensiones de tierras, particularmente en el oriente del Estado (Reporteros Hoy, 2018).

La situación actual, en donde predomina una desarticulación del Sistema Apícola, genera graves daños a los apicultores, qué son realmente los que más sufren esta problemática. A través de una entrevista, un apicultor del poniente del estado, con más de 20 años de experiencia en la apicultura, señala que nunca ha oído hablar de un "Sistema Producto Apícola", ni de un Consejo Apícola, ni nada afín. Afirma que desde siempre ha trabajado de manera individual, que no existe un grupo de personas que velen por su seguridad productiva y económica en estas zonas de Yucatán y percibe una apicultura estancada desde hace años. Reconoce que existen y que ha recibido apoyo del gobierno en algún momento, sin embargo, estos apoyos son sumamente escasos y, en la mayoría de las veces, condicionados. Asegura que no logra identificar ninguna ley, norma o reglamento que los proteja a él ni a su actividad (Apicultor 1, comunicación personal, 10 de octubre de 2019). Otro apicultor de la misma zona con 17 años de experiencia, percibe un grave deterioro de la apicultura en los últimos años, debido a la escasa floración, a la deforestación por agricultura, a la ganadería y a los escasos programas de apoyo gubernamental disponibles. Años atrás, recuerda que la apicultura era más fuerte, porque un apicultor podía dedicarse "de lleno" a la apicultura y sacaba suficientes ganancias para mantener a su familia y continuar con la actividad. Hoy debe combinar la apicultura con actividades de traspatio, con la forestería, la ganadería y con otros trabajos informales para lograr su subsistencia. Señala que es difícil trabajar de manera individual y que recientemente está haciendo todo lo posible para ingresar a una cooperativa apícola relativamente cercana a su localidad, lo que no ha logrado por la poca tecnificación de su producción, siendo rechazada por no cumplir con los más mínimos estándares de calidad y buenas prácticas apícolas. Señala que los apoyos son insuficientes y que no llegan a todos los apicultores en su zona. Además, tiene conocimiento que hay apoyos gubernamentales, pero estos son destinados en su mayoría, a las asociaciones y cooperativas, motivo por el cual busca ser integrante de una de ellas. El segundo apicultor no conoce ninguna ley de protección en materia apícola, tampoco ningún reglamento que siente las bases para la práctica de esta actividad; desafortunadamente tampoco logró identificar a ningún integrante clave en la cadena productiva a la que él, aún sin saber, pertenece (Apicultor 2, comunicación personal, 18 de octubre de 2019).

La situación pareciera repetirse en la región noroeste de Yucatán, en donde un tercer apicultor, con más de 15 años de experiencia, y quien pertenece a una cooperativa de apicultores, identifica a las cooperativas y a las asociaciones apícolas como parte de una "cadena en la venta de miel". Señala que, trabajando de manera colectiva, logra mejores precios en la compra de insumos, ya que estos son adquiridos de manera grupal con un cierto descuento. La venta de su producción es directamente al centro de acopio de su organización, lo que representa un ingreso seguro por su miel, al final de su producción. Sin embargo, el precio que ofrece el centro de acopio sigue siendo bajo y poco justo, aunque en comparación del que ofrecen los "coyotes", es mejor (Apicultor 3, comunicación personal, 26 de octubre de 2019). De hecho, la situación económica de los apicultores se complica, pues en algunos centros de acopio, les "machetean" el precio de su producción; en algunas zonas, el 
kilogramo de miel bajó hasta los 15 pesos (descenso del 60\%). Además, si los productores de la región quieren dejar la miel en el centro de acopio, les pagarían a plazos, es decir, a crédito (El Diario de Yucatán, 2019).

Esta situación, desde el punto de vista de los pequeños productores apícolas, presenta ciertas similitudes, a pesar de provenir de diferentes regiones del Estado de Yucatán. Se puede observar el desconocimiento generalizado del marco normativo que, por medio de leyes, normas o reglas, protege la actividad apícola. Al mismo tiempo, existe el desconocimiento de un sistema que se articule con actores clave de todos los sectores, como es el empresarial, el gubernamental, la sociedad civil, la academia, entre otros, que participen en beneficio de la apicultura. Lo más grave de esto último es que, desde el sector gubernamental, tampoco se tiene plena identificación de este necesario sistema. Un integrante de la SADER en Yucatán, reconoce la existencia de un Sistema Producto Apícola en estas regiones; sin embargo, desconoce si opera como debería. A pesar de su posición, no tiene conocimientos sobre si se están llevando a cabo los trabajos y actividades de regulación, vinculación, protección y fomento, correspondientes a un Sistema Producto Apícola. En contraste, señala que los eslabones de la cadena productiva en las regiones I Poniente y II Noroeste de Yucatán se encuentran dispersos y desorganizados, lo que beneficia únicamente a los comercializadores que poseen el acopio de la miel, y mientras más disperso se encuentre este sistema, más beneficiados se ven éstos últimos, por los precios que les ofertan a los productores, a quienes no les queda otra opción que aceptar el injusto precio. Desde su institución, destaca el funcionamiento de algunos programas de apoyo agropecuario, entre ellos, el Programa de Producción Pecuaria Sustentable y Ordenamiento Ganadero y Apícola (PROGAN), con el cual se apoya al apicultor para mejorar su producción y tecnificarla, desarrollar sus colmenas, manejo de reinas, capacitarse en buenas prácticas y mejorar su equipo. Para obtener este apoyo, que consiste en mejorar la infraestructura de la actividad apícola, el productor o conjunto de productores se acerca a la institución y solicitan lo que necesitan. Comenta que ha percibido una apicultura dispersa a través de los últimos 5 años, y esto puede deberse a la falta de un organismo que regule la actividad apícola, en donde los eslabones condujeran hacia un comercio justo. Asimismo, identifica a 5 grandes empresas en Yucatán que han acaparado el comercio de la miel, y dado el contexto, en donde casi la totalidad de la producción se destina al mercado exterior, esto no permite una organización y un comercio justo para todos los eslabones, ya que estas empresas que han monopolizado el mercado, ponen las reglas del juego. A nivel institucional, reconoce que el objetivo de la SADER es apoyar a la apicultura y lograr que la miel yucateca vuelva a recuperar los niveles de importancia que tenía hace algunos años. A nivel personal, señala que el Sistema Producto Apícola en estas regiones de Yucatán no opera como debería, aún cuando la ley lo impone; esto puede ser debido a que a los integrantes de esta cadena no les conviene que exista una organización en la cadena productiva, porque "los que comercializan y saben de miel" pueden comprar muy barata la miel de los productores a "pie de parcela", por el desconocimiento de los propios productores sobre el tipo de miel que están produciendo. Además, destaca la desorganización del propio apicultor, quien no lleva registros productivos ni económicos de su actividad. Finalmente, concluye que es una urgente necesidad contar con un Sistema Apícola fuerte y sólidamente constituido, capaz de afrontar los intereses ocultos de quienes no quieren que exista esta organización; añade que cualquier tipo de organización generará inconformidad en algún sector, pero esto debe ser eficazmente superado para beneficio de quienes en verdad deberían verse beneficiados, los apicultores (Representante Gubernamental de SADER, comunicación personal, 30 de octubre de 2019).

Al igual que el sector gubernamental, el sector académico es un integrante clave en este sistema. Las investigaciones tanto de carácter científico como de campo, proyectos de conservación, proyectos integrales, así como análisis situacionales en el contexto apícola, son solo algunas de las muchas actividades necesarias que aporta la academia para entender, proteger y fomentar la apicultura. Sin embargo, las insuficientes acciones que parecieran realizarse actualmente, desde la academia hacia la apicultura yucateca, dilucidan un panorama poco alentador. De hecho, un académico opina que la apicultura se encuentra estancada y en peligro de extinción. Reconoce el importante papel que tiene la apicultura para la conservación y regeneración de ecosistemas y resalta el poco fomento, cuidado y apoyo que se le dio a la apicultura en los últimos 5 años; sin embargo, tiene conocimiento de que actualmente el Gobierno, mediante fuertes políticas de conservación y fomento apícola, así como diversos programas gubernamentales, apoyan esta relevante actividad. A través de sus años como catedrático de una simbólica institución de educación superior e investigación en el Estado de Yucatán, se ha percatado de la falta de cultura social y conocimientos sobre la importancia de los servicios ambientales prestados por las abejas; a raíz de esto, impulsado únicamente por su fuerte convicción sobre la importancia de las abejas, crea un equipo interdisciplinario con investigadores ambientales, estudiantes de licenciatura así como de posgrados; ingenieros bioquímicos, entre otros integrados en el proyecto denominado "abeja guardiana" que tiene como objetivo principal el estudio científico de las abejas y la culturalización social de la importancia de cuidarlas así como de preservarlas. Él está convencido de que el Gobierno está en la mejor disposición de apoyar y preservar la apicultura, sin embargo, es necesaria la correcta vinculación entre la sociedad civil, empresas, investigadores ambientales, así como académicos que le propongan al Gobierno, a través de argumentos 
sustentados, el camino correcto a seguir para la preservación de la apicultura. Como investigador y miembro del Sistema Nacional de Investigadores, (SNI), opina que el Sistema Apícola se encuentra en crisis; esto por la simulación y/o replicación de investigaciones que en nada ayudan o aportan a la apicultura ni a la sociedad. Con un fuerte sentimiento de devolverle algo a la sociedad, considera que las problemáticas sociales deben ser atendidas con base a la ciencia y educación; ésta es la única manera de disminuir la violencia, inequidad social, pobreza, desempleo y demás problemas a la sociedad; no se puede seguir elaborando falsos artículos científicos y trabajos de gabinete que no aportan nada a la resolución del problema. Se debe dejar de ver esta situación como una crisis y empezar a verla como una oportunidad de mejorar los problemas reales, con soluciones reales; esto garantizará devolverle a la academia el lugar tan importante que merece en un correcto sistema en donde todos sus integrantes converjan en correcta sinergia (Académico del Tecnológico Nacional de México, comunicación personal, 10 de noviembre de 2019).

Tal como menciona el Académico, con la Sociedad Civil se inicia y termina todo; los empresarios elaboran productos y brindan servicios para la sociedad; la Academia proviene de la sociedad y sus investigaciones deben actuar en pro de ésta, el medio ambiente solo puede ser protegido por la sociedad misma; a través del Gobierno y la legislación vigente, se crean políticas públicas que deben estar alineadas a las demandas de la sociedad; por esto, es menester entender que la sociedad juega el papel más importante en la sustentabilidad de la apicultura. Concientizar a la sociedad e involucrarla en tan complejo sistema, no es tarea fácil; eso lo saben los expertos técnicos, quienes día a día trabajan para lograr este cometido. El experto técnico, quien trabaja concientizando y apoyando a apicultores a lo largo de todo el territorio estatal, opina que la única manera de lograr una verdadera apicultura sostenible es lograr involucrar a todos los integrantes de esta cadena productiva. Con más de 7 años de arduo trabajo en comunidades, ha experimentado todo tipo de resultados; desde apicultores que sólo les interesa recibir dinero o apoyo en especie por parte del Gobierno sin que realmente se comprometan con la actividad, hasta verdaderos casos de éxito que lo llenan de orgullo. Un ejemplo de esto se dio en el Oriente del Estado, en donde una comunidad de apicultores, con el apoyo técnico de este experto apícola, logró conformarse como una sociedad cooperativa que actualmente se auto regula y toma sus propias decisiones de trabajo bajo un equitativo consenso. Además, con una idea clara de sus objetivos, esta cooperativa ha logrado ser reconocida y apoyada por el Gobierno yucateco. A través de programas gubernamentales y el trabajo coordinado con la Comisión Nacional para el Desarrollo de los Pueblos Indígenas, han logrado certificar sus procesos, su producción y comercializarla ellos mismo a través de su tienda oficial, construida y donada por estos dos órganos gubernamentales. Comenta que, como este caso de éxito hay muchos más que son una realidad en el territorio estatal y confía que, con las herramientas necesarias, haya muchos más casos de éxito en el futuro cercano. Actualmente trabaja con comunidades apícolas de los 4 ejes cartesianos en el Estado, dotándolos de herramientas técnicas como son los manuales de buenas prácticas apícolas, cómo combatir las enfermedades que se presentan en los apiarios y cómo lograr una mejor producción; todo lo anterior con un alto grado de concientización y respeto por la actividad, por todo lo que representa la actividad para el beneficio del ser humano. En su opinión personal, cree firmemente que la solución a la problemática apícola está en manos de la misma sociedad, ya que es esta la única responsable de demandar lo necesario para la resolución concreta de sus necesidades. Reconoce que la sociedad está en el centro de todo; de ella salen los integrantes de la academia, del gobierno y de las empresas, pero necesita de las herramientas correctas: educación, cultura, pasión por la naturaleza y un alto grado de calidad humana (Experto Técnico Apícola, comunicación personal, 19 de noviembre de 2019).

Finalmente, pero no menos importante, las empresas. El sector empresarial se conforma por todos los proveedores de insumos y servicios necesarios para la llevar a cabo la práctica apícola. Asimismo, los distribuidores y comercializadores de todos los productos y subproductos provenientes de la colmena forman parte de este sector. Una joven empresaria yucateca de este sector, la cual exporta miel en polvo a países europeos, ha sido testigo de las problemáticas apícolas y del comercio injusto que enfrentan los apicultores en las comunidades rurales; a través de un proyecto que se gestó desde la academia en 2015, ha logrado darle un importante valor agregado a la miel yucateca al procesarla y crear miel en polvo. Teniendo como importante valor empresarial el comercio justo con los apicultores, ha creado políticas empresariales de responsabilidad social: capacitando a apicultores para lograr una producción con buenas prácticas apícolas, mejorando el equipo, así como la indumentaria para la actividad apícola y procurando cuidar la calidad de vida de sus trabajadores y las familias que de ellos dependen. De esta manera, recuerda con gran pesar el largo camino que recorrió en diferentes instancias gubernamentales y organizaciones de índole privada para lograr la aceptación de su proyecto integral, el cual le da la misma importancia al eje económico, como al ambiental y al social; por esto, opina que actualmente el entorno empresarial le da un peso desproporcionado a la dimensión económica sobre la social y la ambiental, situación con la que no está de acuerdo pero no puede cambiar "las reglas del juego". Considera que actualmente el sector empresarial apícola trabaja de manera aislada de los apicultores, limitando las oportunidades que pudiera tener la actividad si se trabajara de manera integral. También destaca 
la descoordinación en el seguimiento de los objetivos y planes de cada Gobierno en turno; esto debido a que, en su experiencia personal, ha tenido convenios de colaboración y alianzas estratégicas con integrantes de la SEDER de Yucatán, con diputados federales en turno y con personal del despacho del Gobernador, a través de vinculaciones efectivas con apicultores que necesitan vender su producción. Sin embargo, al iniciar una nueva administración, y sobre todo de partidos políticos diferentes, estos convenios de colaboración y alianzas estratégicas sufren una desarticulación y se debe iniciar el proceso nuevamente. Lo anterior ha provocado que actualmente la empresaria yucateca no sea capaz de identificar puntualmente a otros integrantes claves de este Sistema Apícola, lo que genera un desconocimiento de las actividades, relaciones y convenios que se están gestando actualmente en este Gobierno en pro de la apicultura. Reconoce que la única manera que asegurar y preservar la práctica apícola en estas 2 regiones de Yucatán es logrando una correcta vinculación entre todos los sectores de este sistema, a través de sus integrantes clave. En su trayectoria profesional y empresarial ha conocido graves problemáticas apícolas, pero confía en que, con proyectos empresariales integrales en donde se respete a todos los integrantes de la cadena apícola a través de un comercio justo y dándole el mismo peso a todas las dimensiones de la sustentabilidad, la apicultura podría ocupar los lugares de importancia que le corresponden (Empresaria Exportadora Apícola, comunicación personal, 5 de diciembre de 2019).

\section{DISCUSIÓN}

Los resultados de la investigación demuestran que actualmente el Sistema Producto Apícola de las regiones I Poniente y II Noroeste del Estado de Yucatán, que debería estar trabajando en correcta sinergia de acuerdo a la ley, se encuentra desarticulado, lo que marca un rumbo desfavorable para la apicultura yucateca en los años venideros. De hecho, a través de la revisión documental se mostró la existencia de la Ley de Desarrollo Rural Sustentable la cual, en su artículo tercero fracción XXXI, categorizan a la actividad apícola como importante actividad agropecuaria generadora de divisas para el país (LDRS, 2001). Por esto, debería existir un Sistema Producto fuerte, capaz de combatir las amenazas externas y superarlas pero, no está sucediendo esto. De acuerdo con los artículos periodísticos y de divulgación que se consultaron para este artículo, existen pocas o nulas alianzas estratégicas entre los actores clave del Sistema a nivel nacional, estatal y regional. De hecho, este tipo de notas periodísticas va en aumento y, de acuerdo con la información que presenta esta investigación, no se está trabajando de manera coordinada para solucionar los problemas de raíz. Lo expuesto por el Representante No Gubernamental del Comité Nacional Sistema Producto demuestra una completa desarticulación entre los integrantes de esta cadena, siendo que no se les ha dado la importancia necesaria a estas representaciones como ejes rectores para la elaboración de las políticas agropecuarias en el país. Este hallazgo refuerza lo descrito por Cuevas, Baca y Aguilar (2011), quienes consideran que la principal desventaja del enfoque de los Sistemas Producto es dejar que un comité de representantes definan, muchas veces con conocimiento limitado, las problemáticas y establezcan las políticas agropecuarias para el país.

Los resultados de esta investigación exhiben 3 grandes problemáticas que afectan gravemente a la apicultura en las regiones I Poniente y II Noroeste del Estado: (1) El reglamento de operación o marco normativo de potección para la apicultura, (2) las políticas públicas que apoyan, fomentan y buscan detonar esta actividad agropecuaria y (3) las relaciones o alianzas estratégicas fundamentales para el correcto funcionamiento de la actividad. A continuación se presenta el análisis de los principales hallazgos de la investigación y se contrastan con algunos resultados de otros estudios, notas periodísticas y artículos de divulgación encontrados a través de la revisión documental.

\section{Reglas}

En México, a través de la Ley de Desarrollo Rural Sustentable, se presenta el fundamento legal acerca de la creación y funcionamiento de los Sistemas Producto en general, incluyendo el Sistema Producto Apícola. En Yucatán, a través de la ley de Protección y Fomento Apícola del Estado de Yucatán, bajo el decreto número 521 publicado el 6 de julio de 2004, se habla de la integración y se puntualiza el funcionamiento de un Comité Sistema Producto Apícola de Yucatán, cuyo principal objetivo es la integración de todos los participantes del sistema para resolver problemáticas apícolas. Tal como lo menciona Miranda (2016), la clave se encuentra en la unión y el trabajo colaborativo de los actores principales que convergen, de manera directa o indirecta, en el Sistema Apícola, quienes deben fortalecer el mismo en todos los niveles, asegurando la búsqueda constante de las mejores soluciones a los desafíos que se presenten. Desafortunadamente, los resultados presentados en esta investigación demuestran que el Sistema Producto Apícola en las regiones I Poniente y II Noroeste de Yucatán se encuentra desarticulado y que sus principales integrantes, quienes son plenamente identificados en el artículo 9 de la Ley de Protección y Fomento Apícola del Estado de Yucatán (Decreto 521/2004, 2004), no logran identificar a otros integrantes del mismo sistema, por ello, desconocen las actividades que se están 
llevando a cabo e implementando en pro de la apicultura yucateca; esto limita considerablemente una correcta vinculación y sinergia entre las actividades que, según la ley anteriormente mencionada, deberían estarse efectuando actualmente para lograr la sostenibilidad de esta actividad productiva de gran importancia para el Estado, siendo que más de un cuarto de todos los apicultores del país se concentran en esta región peninsular. De acuerdo a las notas periodísticas presentadas, no se está respetando ni aplicando la Ley de Protección y Fomento Apícola del Estado de Yucatán, la cual en los decretos 521/2004 y 537/2017 establece claramente el Reglamento de la Ley de Protección y Fomento Apícola del Estado de Yucatán. Los documentos periodísticos exponen retos actuales que no han sido atendidos por las autoridades, una apicultura en crisis y una actividad que dista de llegar a ser sostenible (Güemes et al., 2003). Esta información es respaldada con los resultados de la investigación, en donde los propios apicultores y empresarios inmersos en la producción y comercio del dulce líquido se quejan de una falta de apoyos y estabilidad en el sistema. Además, agravando esta situación, la falta de coordinación entre las instancias públicas estatales y federales, ha provocado una completa desinformación y descoordinación, en donde en más de una ocasión ha traído consecuencias graves para la apicultura yucateca, tal es el caso de la controversial decisión de la Suprema Corte de Justicia de la Nación, cuyas decisiones se contraponen a los intereses de la apicultura en Yucatán, dejando a la vista una completa descoordinación entre el Gobierno Federal y Gobierno Estatal (Santana, 2019).

En este contexto, ante la existencia de un reglamento que pretende proteger y fomentar la actividad apícola en Yucatán, propuesta por el poder ejecutivo y avalada por el poder legislativo del Estado, no debería haber una contraposición con el Poder Judicial de la Federación para que sea derogada. Esto demuestra una desalineación entre el marco normativo estatal, que se decretó precisamente para proteger la apicultura y los intereses de los apicultores yucatecos, y los desconocidos intereses de la Federación por invalidarla. Con acciones como esta, se agrava la desorganización entre los integrantes del Sistema Producto Apícola de las regiones I Poniente y II Noroeste del Estado de Yucatán, generando que estos se sientan amenazados por la descoordinación entre los representantes de justicia del país con los del Estado. Asimismo, este tipo de acciones y decisiones, genera una desvinculación entre las problemáticas y necesidades reales, así como la posible modificación del marco normativo, con nuevas políticas gubernamentales, resultantes de estas infundadas decisiones.

Como menciona Cuevas, Baca y Aguilar (2011), la asociación de intereses mutuos y la agrupación de actores clave a diferentes niveles de gobernabilidad permitirá una mejor gestión y toma de decisiones. Cuando no existen estos intereses mutuos, no se puede asegurar que los integrantes del Comité Sistema Producto Apícola trabajen de manera colaborativa y desinteresada por el bien de la apicultura yucateca ni que tengan la representatividad y aceptación de la cadena productiva.

De esta manera, el marco normativo debe considerar nuevas reglas de operación que doten a los productores de las herramientas humanas y técnicas para que sean capaces de crear nuevos abordajes en materia de innovación hacia su producción; en este momento, no es necesario pensar en tecnologías altamente sofisticadas en la apicultura, bastaría con una correcta capacitación técnica y empresarial, tecnología de nivel medio que logre tecnificar su producción en un nivel por encima de la práctica tradicional y políticas públicas alineadas a apoyar economías locales.

\section{Políticas}

Recientemente, y debido a la necesidad de apoyar técnica y económicamente a la gran cantidad de apicultores que desempeñan esta actividad en el territorio estatal (ver figura 1), el Gobierno del Estado de Yucatán ha implementado fuertes políticas gubernamentales para favorecer, de manera directa e indirecta, a las 7 regiones del Estado, incluyendo las regiones I Poniente y II Noroeste; algunos de los apoyos más importantes que se pudieron identificar son: (1) Fondo de Apoyo a la Productividad Agropecuaria del Estado de Yucatán, en donde se financian proyecto productivos en las actividades agropecuarias y el productor aporta el 10\% del proyecto; (2) PROGAN, el cual otorga subsidios para apoyar diversas actividades agropecuarias; (3) Programa de Desarrollo de Unidades Productivas Campesinas e Insumos Rurales, el cual incentiva la economía rural mediante apoyos permanentes enfocados al desarrollo de productores agropecuarios; (4) Programa Peso a Peso, el cual impulsa actividades agropecuarias, pesqueras y acuícolas mediante fondos de coparticipación y (5) Programa de Apoyo Directo al Desarrollo Rural, el cual busca capitalizar las actividades agropecuarias, pesqueras, acuícolas, agroindustriales o cualquier otra actividad económica del medio rural. 


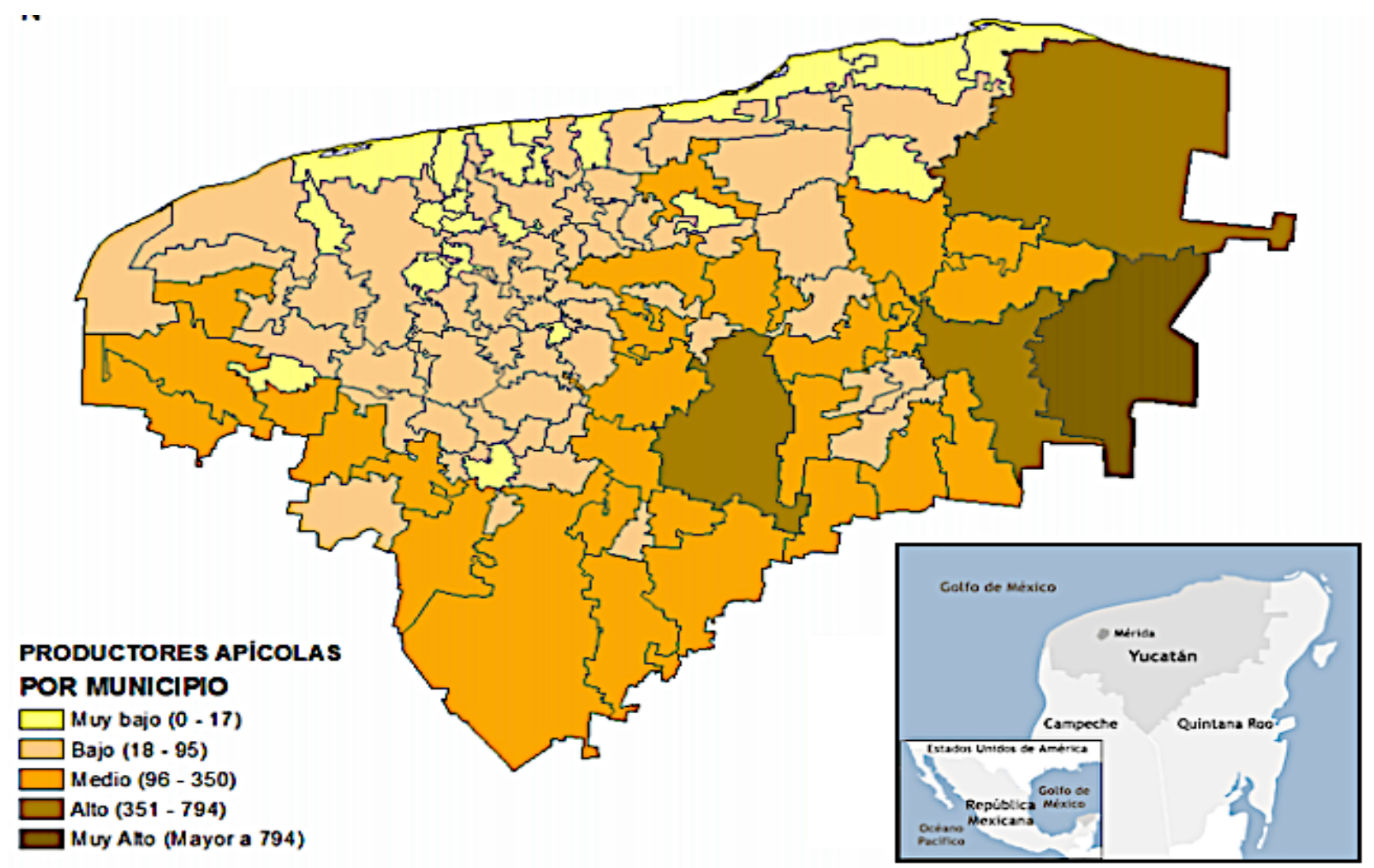

Figura 1. Ubicación de Yucatán a nivel país y sus principales zonas apícolas. Fuente: Elaboración propia.

Estos programas forman parte de una serie de políticas gubernamentales con las que el Gobierno pretende proteger y detonar la actividad productiva apícola; en alineación con lo expuesto por el integrante de la SADER, estos recursos buscan apoyar directamente a los apicultores para resolver sus principales problemas, como son la falta de equipo para la práctica apícola, la falta de liquidez inmediata para afrontar los costos de la producción y la obtención de colmenas. Sin embargo, Merrill-Sands (1984) menciona que los pequeños productores yucatecos siguen una lógica familiar de subsistencia, lo cual indica claramente una continua dependencia de programas públicos, sin los cuales no logran aumentar su producción y, en el peor de los casos, abandonan la actividad. De hecho, resultados de esta investigación demuestran que algunos actores clave del sistema opinan que, aunque estos programas de apoyo tienen buena intención, sus objetivos no se encuentran alineados con la problemática raíz que enfrentan los apicultores; en contraste, muchos de estos apoyos gubernamentales únicamente responden a problemáticas superficiales e inmediatas como falta de liquidez económica. Se considera que algunos programas carecen del apoyo de los demás integrantes del Sistema Apícola; políticas que no poseen las bases metodológicas ni científicas de la academia, que no están dirigidas a proteger ni fomentar un comercio justo con los empresarios y que muchas veces se hacen sin considerar la protección del medio ambiente (Suárez, 2017). Todo esto deriva en que no se resuelven los verdaderos problemas de raíz, únicamente se ofrecen soluciones fugaces que pronto demandarán más apoyos económicos. En este contexto, Güemes et al. (2003) consideran que las actuales políticas encaminadas al comercio internacional muestran una clara desventaja para los pequeños productores del Estado, porque pocas veces conocen y entienden las dinámicas comerciales globales, sus tendencias y sus estructuras, pero se les obliga a participar en ellas, imponiéndoles procesos más eficientes y estándares de calidad más elevados, además de la exigencia constante de darle mayor valor agregado al producto, con el fin de generar más beneficios económicos, por lo tanto, la actual dinámica de comercio global no corresponde a la realidad de la región. En este contexto, es importante subrayar que las políticas gubernamentales deben apoyar a las economías locales para que éstas sean capaces de crecer, autogobernarse y estar claramente alineadas a las necesidades reales de los integrantes del Sistema Apícola, con el objetivo de resolver los problemas reales de los apicultores, de otro modo, esta actividad agropecuaria no logrará su sostenibilidad. 


\section{Relaciones}

A través de cada una de las entrevistas aplicadas, se dilucidó un Sistema Producto Apícola débil y descoordinado, en donde los integrantes claves realizan actividades en pro de la actividad agropecuaria desde sus "trincheras", sin embargo, al desconocer lo que otros integrantes están haciendo, estas actividades se ven limitadas y no logran ser potenciadas por un sistema integral y coordinado. Como se mencionó anteriormente, la Ley de Protección y Fomento Apícola del Estado de Yucatán, publicado desde 2004, puntualiza cómo se debe integrar el Consejo Apícola de Yucatán, pero también, a través del artículo 10 de esta Ley, se plasman las actividades específicas a realizar por este Consejo, entre las que destacan: (1) presentar el programa rector de la apicultura estatal y dar seguimiento permanentemente, (2) integrar, comunicar, organizar y coordinar a los agentes participantes en la actividad apícola, (3) equilibrar la producción con el consumo con el fin de mejorar la competitividad y (4) aumentar el bienestar socioeconómico de los productores apícolas y demás participantes de la cadena de producción (Decreto 521/2004, 2004). En contraste, los resultados de esta investigación demuestran que no existen tales reuniones de integración y, con base a lo expuesto por los sujetos de estudio, actualmente las dinámicas, políticas, reglas y normativas en materia de protección y fomento apícola no son eficientes por el limitado conocimiento que posee el Gobierno Estatal de la realidad del sector; esto ha llevado a tomar decisiones que muchas veces no van acorde a las verdaderas necesidades de la problemática apícola; además, la falta de investigaciones integrales, interdisciplinarias, reales, oportunas y fidedignas generan un visible sistema incapaz de defender a la apicultura yucateca de amenazas externas.

Todo lo anterior resulta grave si se considera que la falta de capacidades humanas se encuentra dada por las pocas alianzas estratégicas y convenios de colaboración que actualmente existen en estas regiones del Estado para afrontar de manera integral e interdisciplinaria las amenazas venideras hacia la apicultura. Se debe entender que la única manera de afrontar estos desafíos es trabajando en equipo, a través del aprendizaje colaborativo y el respeto hacia los conocimientos y tradiciones que cada integrante del sistema puede aportar. Tal como lo menciona Cuevas et al. (2011), se debe aprovechar que la figura de los Sistemas Producto cuentan con un marco normativo legal bajo el cual, es posible demandar enérgicamente la formulación de estrategias de mejora, provenientes de diagnósticos reales, con el fin de generar acciones que impacten de manera directa y puntual las problemáticas reales. De esta manera, se pueden crear planes integrales y dotar a los productores apícolas de las herramientas humanas, técnicas, empresariales y de innovación para mejorar su producción a través de un comercio justo que garantice beneficios para todos los integrantes del sistema.

Finalmente, ante la débil e incipiente existencia de un Sistema Apícola, integrado por actores claves que se involucren y protejan cada una de las etapas de la cadena de producción, la apicultura yucateca de las regiones I Poniente y II Noroeste se ha convertido en una actividad que necesita complementarse con actividades secundarias como la agricultura, animales de traspatio, silvicultura, entre otras. Los resultados de esta investigación demuestran un desinterés por la actividad, lo cual ha provocado que estas regiones de Yucatán entren a una crisis de despoblamiento de abejas y otros polinizadores, poniendo en grave riesgo desde la permanencia de la actividad, la derrama económica generada por el comercio internacional que representa, los servicios ambientales que proporciona, hasta la regeneración y conservación de ciertos ecosistemas (Miranda, 2016). De hecho, Enciso (2018) menciona que los servicios ambientales generados por la producción apícola están en riesgo debido a la introducción de transgénicos en la región sur de México, lo que provoca una reducción en la calidad de la miel y compromete una producción nacional casi destinada en su totalidad al mercado extranjero. En este contexto, y agravando la situación, el Sistema Producto Apícola en las regiones I Poniente y II Noroeste de Yucatán no respeta ni funciona de acuerdo al marco normativo en materia de protección y fomento con el que cuenta la apicultura. Los actores clave integrantes de este Sistema Apícola actualmente no se encuentran preparados para combatir amenazas externas hacia la apicultura yucateca como son la falta de capacidades humanas, de innovación, y políticas públicas, tal y como se expone en los diversos medios periodísticos regionales y nacionales.

\section{REFERENCIAS BIBLIOGRÁFICAS}

AIM. (3 de junio de 2019). La apicultura, en crisis. Diario de Yucatán. https://www.yucatan.com.mx/yucatan/ la-apicultura-en-crisis

Cortés, M., \& León, M. (2004). Generalidades sobre metodología de la investigación. Ciudad del Carmen, Campeche. México D.F.: Universidad Autónoma Del Carmen.

Cuevas, V., Baca, J., \& Aguilar, J. (2011). El concepto de Sistema Producto como eje de las políticas agropecuarias en México. Análisis Del Medio Rural Latinoamericano, 6(57), 83-94. 
Decreto 521/2004. (6 de julio de 2004). Ley de Protección y Fomento Apícola del Estado de Yucatán. Boletín del Estado de Yucatán, 3-12. https://www.poderjudicialyucatan.gob.mx/digestum/marcoLegal/02/2012/DIGESTUM02054.pdf

Decreto 537/2017. (31 de octubre de 2017). Diario Oficial del Gobierno del Estado de Yucatán, 19-38. http:// www.yucatan.gob.mx/docs/diario_oficial/diarios/2017/2017-10-31_2.pdf

Enciso, A. (10 de septiembre 2018). Peligra apicultura en Campeche por soya transgénica. La Jornada, 37. https://www.jornada.com.mx/2018/09/10/sociedad/037n1soc

Güemes, F., Echazarreta, C., Villanueva, R., Pat, J., \& Gómez, R. (2003). La apicultura en la península de Yucatán: Actividad de subsistencia en un entorno globalizado. Revista Mexicana del Caribe, 8(16), 117-132.

Ley de Desarrollo Rural Sustentable. (1 de enero de 2001). Diario Oficial de la Federación, 47-48. http://www. diputados.gob.mx/LeyesBiblio/pdf/235_120419.pdf

Martínez-Salgado, C. (2012). El muestreo en investigación cualitativa: principios básicos y algunas controversias. Ciencia y Saúde Coletiva, 17(3), 613-619.

Merrill-Sands, D. (1984). The Mixed subsistence-commercial Production System in the Peasant Economy of Yucatán, México: An Anthropological Study in Commercial Beekeeping (Tesis doctoral). Cornell University, New York.

Miranda, F. (26 de agosto de 2016). México, al borde de crisis por despoblamiento de abejas. Milenio. https:// www.milenio.com/estados/mexico-al-borde-de-crisis-por-despoblamiento-de-abejas

Santana, R. (15 de agosto de 2019). La SCJN invalida decreto que declaraba zona libre de transgénicos a Yucatán. Proceso. https://www.proceso.com.mx/596222/la-scjn-invalida-decreto-que-declaraba-zona-libre-de-transgenicos-a-yucatan

Secretaría de Desarrollo Agropecuario del Estado de México. (13 de septiembre de 2017). Sistemas Producto. http://sedagro.edomex.gob.mx/sistemas_producto

Servicio de Información Agroalimentaria y Pesquera [SIAP]. (enero de 2020). Avance mensual de la producción pecuaria 2020. http://infosiap.siap.gob.mx/repoAvance_siap_gb/pecAvanceProd.jsp

Suárez, G. (15 de octubre de 2017). Apicultura en la Península de Yucatán: vivir de la selva de forma sostenible. Consejo Civil Mexicano para la Silvicultura Sostenible. https://www.ccmss.org.mx/apicultura-peninsula-yucatan-miel-sostenible-selvas-mexico/

Yin, R. (1989). Case Study Research: Design and Methods, Applied social research Methods Series. Newbury Park, CA: Sage.

Zapata, T. (19 de abril de 2018). Exponen retos y problemáticas de la apicultura en Yucatán. Reporteros Hoy. https://reporteroshoy.mx/wp/exponen-retos-y-problematicas-de-la-apicultura-en-yucatan.html

Correo electrónico: joacofranco200294@gmail.com 